\title{
Thermosensitive molecularly imprinted polymers on porous carriers: Preparation, characterization and properties as novel adsorbents for bisphenol A
}

\author{
Ruichen Dong ${ }^{\mathrm{a}, \mathrm{b}}$, Jinhua $\mathrm{Li}^{\mathrm{b}}$, Hua Xiong ${ }^{\mathrm{a}, *}$, Wenhui Lu ${ }^{\mathrm{b}}$, Hailong Peng ${ }^{\mathrm{a}}$, Lingxin Chen ${ }^{\mathrm{b}, * *}$ \\ a State Key Laboratory of Food Science and Technology, Nanchang University, Nanchang 330047, China \\ ${ }^{\mathrm{b}}$ Key Laboratory of Coastal Environmental Processes and Ecological Remediation, Yantai Institute of Coastal Zone Research, Chinese Academy of Sciences, \\ Yantai 264003, China
}

\section{A R T I C L E I N F O}

\section{Article history:}

Received 27 April 2014

Received in revised form

22 June 2014

Accepted 25 June 2014

Available online 6 July 2014

\section{Keywords:}

Molecularly imprinted polymers

Thermosensitive polymers

Bisphenol A

Surface imprinting

\begin{abstract}
A B S T R A C T
Thermosensitive molecularly imprinted polymers (T-MIPs) on porous carriers were prepared via the synergy of dual functional monomers of 4-vinylpyridine (VP) and N-isopropylacrylamide (NIPAM), for selective recognition and controlled adsorption and release of bisphenol A (BPA) by the temperature regulation. The porous polymer supporter was synthesized by multistep swelling of polystyrene and then both the NIPAM with temperature responsiveness and the basic monomers of VP were grafted on them in a simple way. The resultant T-MIPs showed high binding capacity, fast kinetics, and the adsorption processes were found to follow Langmuir-Freundlich isotherm and pseudo-second-order kinetic models. The adsorption capacity increased slightly along with the rise of temperature (such as $20{ }^{\circ} \mathrm{C}$ ) under lower critical solution temperature (LCST, $33^{\circ} \mathrm{C}$ ) and decreased fast above LCST (such as $50{ }^{\circ} \mathrm{C}$ ). Subsequently, the T-MIPs were employed as novel adsorbents for selective solid-phase extraction (SPE) of BPA from seawater and yogurt samples. Satisfying recoveries in the range of 94.83-98.47\% were obtained with the precision of $3.21 \%$ at ambient temperature $\left(20^{\circ} \mathrm{C}\right)$. Through 6 adsorption-desorption cycles, the reusable T-MIPs exhibited a good recoverability with the relative standard error within $9.8 \%$. The smart T-MIPs provided great potentials for selective identification, adsorption/release and removal of BPA by simple stimuli responsive regulation.
\end{abstract}

(c) 2014 Elsevier B.V. All rights reserved.

\section{Introduction}

Phenolic environmental estrogens (PEEs) have received considerable concerns in the field of pollution monitoring and remediation, which may change the function of the endocrine systems and consequently elicit negative health effects $[1,2]$. Bisphenol A (BPA), one of PEEs, has been widely used as raw material of plastics and resins, antioxidants and polymerization inhibitors [3,4]. However, prolonged utilization and abuse of BPA leads to its accumulation in environment and thereafter causes environmental and health threats [3-5]. Some hazards and adverse influences have been identified in model animals exposed to low doses of BPA even much lower than the regulated safe dose levels [5]. Meanwhile, complicated matrix effects make the residue analysis of BPA face crucial challenges [6]. So, it is urgently required to develop simple, fast and

\footnotetext{
* Corresponding author. Tel./fax: +867916634810.

*** Corresponding author. Tel./fax: + 865352109130.

E-mail addresses: huaxiong100@126.com (H. Xiong), lxchen@yic.ac.cn (L. Chen).
}

high sensitive methods for identifying the presence and determining the levels of BPA.

The most frequently used methods for separation and determination of BPA mainly include high performance liquid chromatography (HPLC), HPLC-mass spectrometry (MS), and gas chromatography-MS (GC-MS), generally following suitable sample pretreatment processes such as the widely used liquid-liquid extraction (LLE) and solid-phase extraction (SPE) [7-11]. The main problem associated with traditional sorbents of SPE is the low selectivity and thereby low adsorption capacity and enrichment efficiency. Therefore, developing specific materials with high selectivity is necessary. Molecularly imprinted polymers (MIPs) are gaining popularity [12]. For example, Wang et al. synthesized MIPs via ring-opening metathesis polymerization [13] and Li et al. prepared hollow porous MIPs using tetrabromobisphenol A as dummy template [14], which were both used as SPE sorbents to enrich BPA combining with HPLC-UV determination.

Besides, another promising material, stimuli-responsive polymers (SRPs), also known as environmental responsive polymers or smart polymers, has also attracted widespread interest $[15,16]$. SRPs are able to respond to specific external stimuli with considerable 
changes in their physicochemical properties, including molecular chain structure, solubility, surface structure, swelling or dissociation behavior, etc., and various stimuli signals are available such as temperature, $\mathrm{pH}$, magnetism and light [17]. By combining MIPs with SRPs, the resultant seductive functional materials, stimuli responsive MIPs (SR-MIPs) have been developed, occupying the advantages of molecular recognition ability for template/target species and responsive ability to external stimuli [17-19]. That is, the release and adsorption of template/target molecules can be achieved through external stimuli regulation. Excitedly, a number of SR-MIPs have been designed and synthesized for recognition and detection of BPA [17,20-23]. For instance, Griffete et al. presented inverse opals of imprinted hydrogels for the detection of BPA and displayed large responses to external $\mathrm{pH}$ stimuli related to the thickness of hydrogel film [20]. Liu et al. described a novel core-shell BPA imprinted nanoparticle by polychloromethylstyrene coated superparamagnetic material via surface initiated atom transfer radical polymerization [21]. Lin et al. adopted bisphenol $\mathrm{F}$ as a dummy template to prepare MIPs for BPA with a magnetic supporter which could increase the extraction efficiency [22]. Lu et al. fabricated a photoelectrochemical (PEC) sensor to detect BPA based on vertically aligned $\mathrm{TiO}_{2}$ nanotubes with surface molecularly imprinted polypyrrole, and a highly sensitive PEC response for BPA was obtained [23]. It is found that magnetic SR-MIPs are mostly discussed; other type ones are rarely mentioned. Meanwhile, to the best of our knowledge, temperature responsive SR-MIPs have never been reported for BPA till now.

$\mathrm{N}$-Isopropylacrylamide (NIPAM) is a well-known intelligent material that is responsive to the temperature, which has the property of a soluble (hydrophilic)-insoluble (hydrophobic) transition at a lower critical solution temperature (LCST) of about $33^{\circ} \mathrm{C}$ [24]. It displays a coil (soluble) state when the solution temperature is below the LCST, as well as a collapsed (insoluble) state at above the LCST [24]. The thermally responsive behavior of NIPAM can be used to design smart MIPs; this may be the ideal way to controlled adsorption and release of template molecules according to temperature changes.

In this work, we firstly synthesized the smart material of thermosensitive MIPs (T-MIPs) by using NIPAM as temperature responsive functional monomer, for selective recognition and extraction of BPA from real water and food samples. A simple synthesis protocol was used by grafting two types of functional monomers on porous polymers carrier, and plenty of hollow pores were beneficial for mass transfer. The synergy effect of dual monomers of NIPAM and 4-vinylpyridine (VP) would contribute greatly to the performances of T-MIPs. Binding property, imprinting ability and temperature-regulated behavior were systematically investigated. Accordingly, the obtained smart T-MIPs were successfully applied to SPE for BPA in seawater and yogurt samples, indicating great potential for the analysis/removal of the PEEs in complicated matrices.

\section{Experimental}

\subsection{Materials and instruments}

Poly-vinylpyrrolidone (PVP), poly-vinylalcohol (PVA), $\mathrm{N}$-isopropylacrylamide (NIPAM, 98\%) and N,N'-methylenebis(acrylamide) (MBAA, 99\%) were purchased from Aladdin (Shanghai, China). Ethylene glycol dimethacrylate (EGDMA, 98\%), divinylbenzene (DVB, 80\%), styrene (99\%), thioglycolic acid, 2,2-bis(4-hydroxyphenyl) (BPA), $17 \beta$-estradiol ( $\beta$-E2, 99\%), phenol (PE, 99.6\%) and cholesterol (CE) were obtained from Aladdin (Shanghai, China). Estriol (E3, 99.5\%), estrone (ET, 99\%), 4-vinylpyridine (VP, 95\%) and methacrylic acid (MAA, 99\%) were provided by Sigma-Aldrich (Shanghai, China). Acetonitrile (ACN, 99.9\%) was purchased from
J\&K Scientific Ltd. (Beijing, China). Dibutyl phthalate (DBP), benzoyl peroxide (BPO, CP), toluene and dichloromethane were purchased from Sinopharm Chemical Reagent Co. Ltd. (Shanghai, China). Dimethylbenzene, absolute ethanol, methanol and sodium dodecyl sulfate (SDS) were attained from Tianjin Reagent Plant (Tianjin, China). Potassium persulfate (KPS) and 2,2-azobisisobutyronitrile (AIBN) were purchased from Shanghai Chemical Plant (Shanghai, China). DVB and MAA were distilled in vacuum to remove stabilizers and AIBN was refluxed over sodium and then distilled. All reagents were of analytical grade and used directly without further purification unless otherwise specified. Aqueous solutions throughout the work were prepared using doubly purified deionized water, which was produced by a Cascada TM LS Ultrapure water system with the water outlet operating at 18.2 $\mathrm{M} \Omega \mathrm{cm}$ (Pall Corp., USA).

The microstructure and elaborated morphology evaluation were examined by an inverted microscope (XDS-1B), a scanning electron microscope (SEM, Hitachi S-4800FE-SEM, $3 \mathrm{kV}$; samples dispersed in ethanol and adding a drop on an aluminum sheet followed by sputter-coated with gold for $85 \mathrm{~s}$ under high vacuum) and a transmission electron microscope (TEM, JEOL, JEM-1400, samples dispersed in ethanol and adding a drop on a copper grid). The particle sizes were measured by Malvern Zetasizer (Nano ZS90, dispersed in absolute ethanol). The chemical component and characteristic functional groups were measured with a thermal gravimetric analyzer (TGA, Mettler $5 \mathrm{MP}, 800{ }^{\circ} \mathrm{C}, 10^{\circ} \mathrm{C} / \mathrm{min}$, nitrogen $50.0 \mathrm{~mL} / \mathrm{min}$ ) and a Fourier transform infrared spectrometer (FT-IR, Nicolet iS10, deduct air background), respectively. The information of specific surface area and pore size was detected by a specific surface and pore size analysis instrument (3H-2000PS4, Beijing). UV-vis spectra were recorded using a Thermo Scientific spectrophotometer (NanoDrop 2000/2000c, USA). Analytes were determined by high performance liquid chromatography (HPLC, Skyray LC-310, China). And HPLC conditions were employed for BPA: mobile phase, $\mathrm{ACN} / \mathrm{H}_{2} \mathrm{O}(90: 10, \mathrm{v} / \mathrm{v})$; flow rate, $1.0 \mathrm{~mL} / \mathrm{min}$; column temperature, $20^{\circ} \mathrm{C}$; UV detection, at $226 \mathrm{~nm}$; injection volume, $20 \mu \mathrm{L}$; analytical column, $\mathrm{C}_{18}$ column with $250 \mathrm{~mm} \times 4.6 \mathrm{~mm}(5 \mu \mathrm{m}$ particle, Waters, USA).

\subsection{Synthesis of polystyrene (PS) seed particles}

PS was synthesized according to the classical dispersion polymerization method [25] with a little modification. Briefly, anhydrous ethanol $(100 \mathrm{~mL})$, deionized water $(20 \mathrm{~mL})$ and PVP $(0.7 \mathrm{~g})$ were added to a $250 \mathrm{~mL}$ three-necked flask and then dispersed evenly by ultrasound and vigorous stirring. Then $5 \mathrm{~mL}$ styrene was added to the above solution. After pouring with nitrogen for $30 \mathrm{~min}$, AIBN (40 mg) was added to the mixture and then heated to $60{ }^{\circ} \mathrm{C}$ and polymerized for $24 \mathrm{~h}$ under nitrogen atmosphere. The obtained PS seed particles were washed five times with ethanol and dried to constant weight under vacuum at $40{ }^{\circ} \mathrm{C}$.

\subsection{Synthesis of hollow porous polystyrene particles (HPS)}

HPS was prepared by a three-step swelling polymerization method [26] with necessary modification. Typically, SDS (0.125 g) and deionized water $(50 \mathrm{~mL})$ were added to a $100 \mathrm{~mL}$ two-necked flask. Then PS $(0.2 \mathrm{~g})$ was added to the above solutions and formed a homogenous emulsion by ultrasound and stirring. Firstly, DBP $(0.7 \mathrm{~mL})$ was added to the emulsion and was stirred for $24 \mathrm{~h}$ at room temperature; secondly, toluene $(10 \mathrm{~mL})$ and MAA ( $4 \mathrm{mmol})$ were added to the solutions and kept for swelling for $24 \mathrm{~h}$; thirdly, DVB $(4.0 \mathrm{~mL})$ and BPO $(60 \mathrm{mg})$ were added to the emulsion, stirring for $24 \mathrm{~h}$, and the temperature of the emulsion was raised to $70{ }^{\circ} \mathrm{C}$ and the reaction was performed for another $24 \mathrm{~h}$ under nitrogen atmosphere. Finally, the HPS particles were obtained by 
centrifuging and washing with dichloromethane and methanol for several times.

\subsection{Preparation of thermosensitive porous polymers imprinted with BPA (T-MIPs)}

Functional monomers were firstly pre-assembled for $6 \mathrm{~h}$ to generate synergistic effect with the addition of cross-linker (MBAA) and initiator (KPS), and two types of co-monomers of NIPAM-MAA and NIPAM-VP were synthesized for comparison. The synthesis process of T-MIPs is described as follows. HPS (100 mg) and PVA $(50 \mu \mathrm{L}$, stabilizer and surface charge modifier) were dispersed in solvent $(40 \mathrm{~mL} \mathrm{ACN}$ and $10 \mathrm{~mL}$ methanol) in a $100 \mathrm{~mL}$ three-necked flask and stirred for $6 \mathrm{~h}$. At the same time, the compound monomers $(4 \mathrm{mmol})$ and template molecule (BPA, $1 \mathrm{mmol}$ ) were mixed to pre-polymerize for $6 \mathrm{~h}$ in a small roundbottom flask. Then the monomer-template compound was added to the HPS solution in three-necked flask, and cross-linker (20 mmol EGDMA/DVB) was added subsequently under vigorous stirring. After blowing nitrogen for $30 \mathrm{~min}$, the solution was heated to $60{ }^{\circ} \mathrm{C}$ and maintained at constant temperature, and then AIBN (20 mg) was added; precipitation polymerization was performed for $24 \mathrm{~h}$ under nitrogen flow. Then the resultant products were filtrated and washed with acetone and methanol several times and the template was eluted by Soxhlet extraction refluxing with methanol/acetic acid $(4: 1, \mathrm{v} / \mathrm{v})$ for $12 \mathrm{~h}$ and methanol/acetic $(9: 1, v / v)$ for another $12 \mathrm{~h}$ until the template was unable to be detected, and the obtained T-MIPs were dried and collected. Meanwhile, the preparation of thermosensitive non-imprinted polymers (T-NIPs) followed the same procedure but without adding the template BPA.

\subsection{Static and kinetic adsorption experiments}

The binding capacity and kinetics properties of T-MIPs and T-NIPs for BPA were determined as follows. Typically, BPA dissolved in $A C N$ of $200 \mathrm{mg} / \mathrm{L}$ was first prepared, and then the solution was diluted to different concentrations with phosphate buffer solution for detection. $10 \mathrm{mg}$ polymers were dispersed in a $5 \mathrm{~mL}$ flask containing $2.0 \mathrm{~mL}$ BPA solutions of various concentrations (1-100 $\mathrm{mg} / \mathrm{L}, 20^{\circ} \mathrm{C}, \mathrm{pH} 7.0$ ), after shaking $24 \mathrm{~h}$, the mixtures were centrifuged and the supernatants were collected by $0.45 \mu \mathrm{m}$ Millipore microfiltration membrane, concentrations of which were determined using HPLC-UV. The binding amounts of BPA are determined by subtracting the residual amounts in solution from initial amounts according to the following equation:

$Q_{\mathrm{e}}=\frac{\left(C_{0}-C_{\mathrm{e}}\right) V}{W}$

where $Q_{\mathrm{e}}(\mathrm{mg} / \mathrm{g})$ is the binding capacity of polymers; $C_{0}$ and $C_{\mathrm{e}}$ $(\mathrm{mg} / \mathrm{L})$ are the initial and equilibrium concentrations, respectively; $V(\mathrm{~mL})$ is the volume of the solutions; and $W(\mathrm{~g})$ is the mass of the polymers added to the solutions.

Meanwhile, the adsorption kinetics was studied by monitoring the temporal amounts of BPA in the solutions of different incubation times $\left(2-240 \mathrm{~min}, 20^{\circ} \mathrm{C}, \mathrm{pH} 7.0, C_{(\mathrm{BPA})}=50 \mathrm{mg} / \mathrm{L}\right)$, calculated by the following equation:

$Q_{t}=\frac{\left(C_{0}-C\right) V}{W}$

where $Q_{e}$ and $Q_{t}(\mathrm{mg} / \mathrm{g})$ are the polymers' equilibrium and temporal binding capacities of BPA, respectively; $C_{0}, C_{\mathrm{e}}$ and $C(\mathrm{mg} / \mathrm{L})$ are the initial, equilibrium and temporal adsorption concentrations in the solutions, respectively; $V(\mathrm{~mL})$ is the volume of the solution; and $W(\mathrm{~g})$ is the mass of polymers added to the solutions. The imprinting factor was defined as $\alpha=Q_{\text {MIP }} / Q_{\text {NIP. }}$ Each group of experiments was carried out five times in parallel.

Moreover, related descriptions on the adsorption isotherms and binding kinetics fitting analyses were given in Supporting information, i.e., Langmuir, Freundlich, Scatchard, Langmuir-Freundlich models $[27,28]$, and pseudo-first-order, pseudo-second-order, Elovich and intraparticle diffusion models [29,30].

\subsection{Thermosensitive property and selectivity experiments}

The temperature sensitivity evaluation of T-MIPs and T-NIPs was performed as follows. $10 \mathrm{mg}$ polymers were dispersed in a $5 \mathrm{~mL}$ flask containing $2.0 \mathrm{~mL}$ BPA solutions of different concentrations $(1,10,30,50,100 \mathrm{mg} / \mathrm{L})$, and then they were immediately transferred to 20,33 and $50{ }^{\circ} \mathrm{C}$ thermostat water baths, and incubated for $24 \mathrm{~h}$. Besides, the influence of solution $\mathrm{pH}$ on the adsorption capacity was studied by measuring the binding amounts at different solution $\mathrm{pH}$ values $(5.8,6.0,6.5,7.0,7.5$ and 8.0 , adjust by corresponding medium PBS, $24 \mathrm{~h}, C_{\mathrm{BPA}}=10$ and $50 \mathrm{mg} / \mathrm{L}$ )

At the same time, selectivity experiments were carried out by using $\beta$-E2, E3, ET, PE and CE as structural analogs. $10 \mathrm{mg}$ polymers were added to $5 \mathrm{~mL}$ flasks containing $50 \mathrm{mg} / \mathrm{L}$ BPA and five structural analogs $\left(20^{\circ} \mathrm{C}, \mathrm{pH} 7.0\right)$, and then the solutions were incubated for $24 \mathrm{~h}$. Finally, the solution was centrifuged, filtrated and determined. The selectivity factor was defined as $\beta=Q_{\text {template }}$

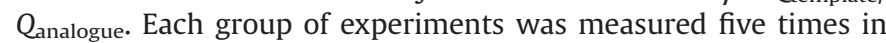
parallel.

\subsection{Preparation of standards and samples}

Stock solutions containing $100 \mathrm{mg}$ of each BPA per liter were prepared by dissolving the required amounts of the standards in ACN. Working solutions were prepared by diluting the stock solutions with appropriate amounts of doubly purified deionized water. They were stored in a refrigerator at $4{ }^{\circ} \mathrm{C}$ before use.

Surface seawater samples were randomly collected from the seaside of the Yellow Sea located in the coastal zone area of Yantai City. Yogurt samples were purchased from a local market. The simple treatment was briefly described as follows: $38 \mathrm{~mL} \mathrm{ACN}$ and $2 \mathrm{~mL}$ seawater/yogurt samples were added to a $50 \mathrm{~mL}$ flask, followed by ultrasonic treatment for $30 \mathrm{~min}$, and then the solutions were filtered by $0.45 \mu \mathrm{m}$ Millipore membranes for use. For spiked tests, appropriate amounts of BPA standards were added into the treated seawater and yogurt samples.

\subsection{SPE procedure}

The T-MIPs were used as the packing materials of SPE column at $20{ }^{\circ} \mathrm{C}$. The extraction process was briefly described as follows. $200 \mathrm{mg}$ polymers were filled in the SPE column $(9.0 \mathrm{~mm}, 3 \mathrm{~mL})$ and compressed. $5 \mathrm{~mL}$ methanol was added for activating the column at the flow rate of $1.0 \mathrm{~mL} / \mathrm{min}$. Then $2 \mathrm{~mL} \mathrm{ACN}$ was added to wash the column followed by loading $1 \mathrm{~mL}$ sample, at the flow rate of $0.2 \mathrm{~mL} / \mathrm{min}$. After washing the column with $2 \mathrm{~mL}$ methanol and deionized water, at the flow rate of $1.0 \mathrm{~mL} / \mathrm{min}$, the adsorbed BPA was eluted with $1 \mathrm{~mL}$ methanol/acetic acid (v/v, 9:1), at $0.2 \mathrm{~mL} / \mathrm{min}$. Finally, the methanol/acetic acid solvents were blown-dried and then the residue was dissolved with ACN to $1 \mathrm{~mL}$, and the amount of BPA was determined by HPLC-UV and the recovery was calculated. Each group of experiments was measured three times in parallel under room temperature. 


\subsection{Reusability of T-MIPS}

The reusability of T-MIPs was measured by washing the packing column repeatedly with methanol/acetic acid (v/v, 9:1) and ACN solvents, and then the column was reactivated by methanol for repetitive use. The spiked solutions containing $5 \mathrm{mg} / \mathrm{L}$ BPA were extracted by the same column for repeating six or more times, and then the recovery was calculated.

\section{Results and discussion}

\subsection{Preparation of T-MIPs for BPA}

T-MIPs were synthesized using two types of functional monomers via their synergistic effect, on a porous carrier material. The preparation process and possible imprinting mechanism are schematically illustrated in Fig. 1 and the condition optimization recipes are listed in Table S1. HPS nanoparticles were first prepared by dispersion polymerization followed by the thermosensitive imprinted polymers grafted onto the cavity in the carrier. Meanwhile, four groups of monomers (NIPAM-VP, NIPAM-MAA, VP, MAA), three crosslinkers (EGDMA, DVB, EGDMA-DVB) and four solvents (ACN-methanol, acetone, chloroform, toluene) were used as the reaction variables (Table S1). As shown in Table S2, dispersion polymerization was selected for HPS, and precipitation polymerization was chosen for the T-MIPs preparation. The results in Table S1 showed that the optimal preparation conditions for T-MIPs were as follows: NIPAM (thermosensitive monomer) coupled with VP as the dual functional monomers $(4 \mathrm{mmol})$, EGDMA as crosslinker $(20 \mathrm{mmol})$ and ACN/methanol $(4: 1, \mathrm{v} / \mathrm{v}$, $50 \mathrm{~mL}$ ) as solvents. As is well known that NIPAM is hydrophilic and presents stretched state below the LCST due to the hydrogen bonding interactions between the amide chains and solution, as well as it is hydrophobic and exhibits collapsed state above the LCST. So, the obtained T-MIPs can adsorb and release BPA by regulating temperature related to LCST, as shown in Fig. 1.

\subsection{Characterization of T-MIPS}

Fig. 2 shows the SEM and TEM images of particles. As can be seen, the PS, HPS and T-MIPs are regular, monodisperse and homogeneous microspheres, and the porous T-MIPs are about $1 \mu \mathrm{m}$ in diameter. Meanwhile, the microscope images in Fig. S1 matched well with Fig. 2. However, the polymer morphologies were quite different and irregular for the T-MIPs using different crosslinkers (T-MIPs-C), different solvents (T-MIPs-S) and the T-NIPs. As shown in Fig. S2a, the T-MIPs-C polymers are crosslinked and conglomerated in knots which may be caused by the crosslinker DVB reacting with HPS carrier, or the cross-link effect is too strong. As seen from Fig. S2b, the T-MIPs-S exhibited irregular spherical morphology, which was likely owing to the decomposition of HPS carrier in improper solvents. As displayed in Fig. S2c, T-NIPs showed some microspheres with little polymers grafted on them, suggesting the polymerization efficiency was very poor. On the other hand, the average particle sizes of PS, HPS, T-MIPs and T-NIPs were roughly measured with Malvern Zetasizer, as shown in Fig. S3, to be 755, 842, 1235 and $1240 \mathrm{~nm}$, respectively, with the precision lower than $65 \mathrm{~nm}$.

The $\mathrm{N}_{2}$ adsorption-desorption isotherms and pore diameter distribution plots of T-MIPs and T-NIPs are shown in Fig. S4. The adsorption and desorption volumes of T-MIPs were higher than those of T-NIPs, as observed in Fig. S4a, and the specific surface area $\left(80.55 \mathrm{~m}^{2} / \mathrm{g}\right)$ was larger than the latter $\left(28.75 \mathrm{~m}^{2} / \mathrm{g}\right)$

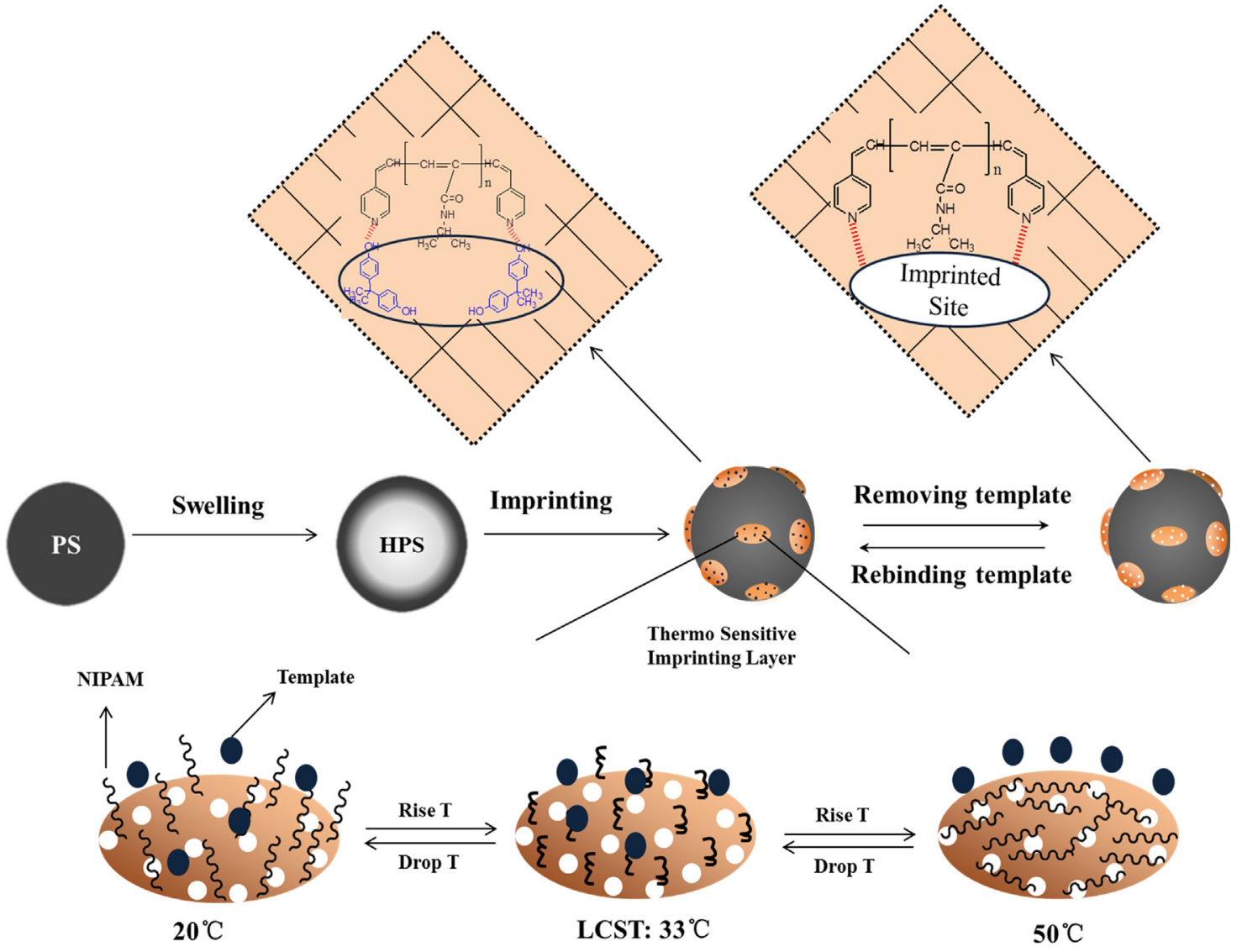

Fig. 1. Schematic illustration of the preparation process and principles of T-MIPs. 

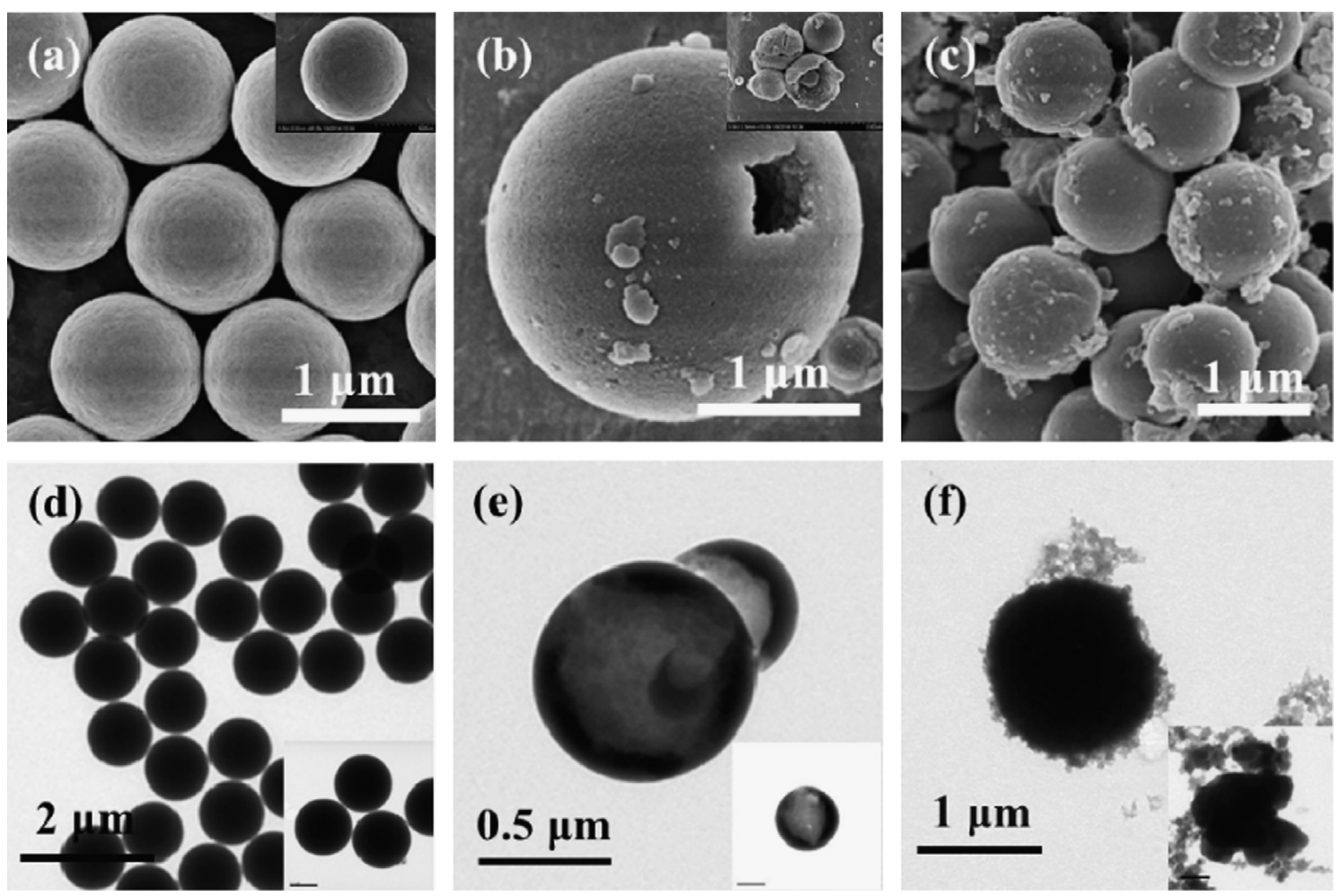

Fig. 2. SEM images of PS (a), HPS (b) and T-MIPs (c) and TEM images of PS (d), HPS (e) and T-MIPs (f).

(Table S3), which indicated that the T-MIPs had more uniform and regular spherical morphologies with well-defined mesoporous structures. Also, it was found when the relative pressure $P / P_{0}$ was less than 0.8 , the curves displayed very low slope values, suggesting that very little amounts of small size pores were present on the surface of T-MIPs. By contrast, when the relative pressure $P / P_{0}$ was higher than 0.8 , the slope values of the curves increased sharply. As shown, the desorption curve was closer but leveled above the adsorption curve, which could be the evidence of a small quantity of well-defined mesoporous/microporus structures existent on the T-MIPs [31]. The T-MIPs exhibited wider pore diameter distribution and larger average pore diameter than T-NIPs, as shown in Fig. S4b, and the cumulative pore volume and average pore diameter were $0.4776 \mathrm{~mL} / \mathrm{g}$ and $22.20 \mathrm{~nm}$, respectively, higher than the T-NIPs $(0.0994 \mathrm{~mL} / \mathrm{g}$ and $11.14 \mathrm{~nm})$, as listed in Table S3. All the results indicated that the large specific surface area and pore diameter of T-MIPs matrix played an important role in the polymerization process and contributed greatly to the adsorption capacity.

The TG analysis curves of the PS, HPS, T-MIPs and T-NIPs are displayed in Fig. S5a reflecting the percentage of weight loss, along with the DTG curves in Fig. S5b describing the weight decrease rate with the time increasing. The corresponding TG parameters are listed in Table S4. As shown, PS presented a smooth TG curve with $94.78 \%$ weight loss, and the maximum loss rate was $1.67 \mathrm{mg} / \mathrm{min}$ at $410{ }^{\circ} \mathrm{C}$, indicating PS was pure and it underwent decomposition at around $400{ }^{\circ} \mathrm{C}$. The range of decomposition temperature became wider for the TG curve of HPS, and its maximum loss rate was $0.044 \mathrm{mg} / \mathrm{min}$ at $464{ }^{\circ} \mathrm{C}$, proving the formation of porous structure in the swelling process. The TG curves of T-MIPs and T-NIPs were similar, in which the maximum loss rate was $0.97 \mathrm{mg} / \mathrm{min}$ at $450.48{ }^{\circ} \mathrm{C}$ and $0.96 \mathrm{mg} / \mathrm{min}$ at $450.46{ }^{\circ} \mathrm{C}$, respectively, and the weight lost by T-MIPs was $82.66 \%$ and by T-NIPs was $79.95 \%$. All these results illustrated the fact that the smart polymers were successfully grafted onto the porous carrier for T-MIPs.
Fig. S5c shows the FT-IR spectra of PS, HPS, T-MIPs and T-NIPs. As shown, for PS, the characteristic absorption peaks at 3025, 3062 and $3081 \mathrm{~cm}^{-1}$ could be ascribed to the stretching vibration of $\mathrm{C}-\mathrm{H}$ bond in the benzene ring, and the strong absorption at 2844 and $2925 \mathrm{~cm}^{-1}$ could be attributed to the stretching vibration of saturated $\mathrm{C}-\mathrm{H}$ bond. In addition, the peaks at 1492 and $1448 \mathrm{~cm}^{-1}$ could be the bending vibration of saturated $\mathrm{C}-\mathrm{H}$ bond in alkane, the absorption at $1598 \mathrm{~cm}^{-1}$ was possibly caused by the benzene ring structure, and the peaks at 757 and $701 \mathrm{~cm}^{-1}$ were the bending vibration of benzene ring substituent. The main characteristic absorption peaks of HPS were the same as PS; however, the absorption peaks at around $3000 \mathrm{~cm}^{-1}$ disappeared, which was very likely due to the substitution or reaction of benzene ring in the swelling process. Two stronger absorption peaks, at $1729 \mathrm{~cm}^{-1}$ being possibly the stretching vibration of $\mathrm{C}=\mathrm{O}$ bond and at $987 \mathrm{~cm}^{-1}$ being the out-plane bending vibration of $\mathrm{C}-\mathrm{H}$ bond in alkene, indicated that PS participated in the addition and oxidation reactions with pore-foaming agents. Interestingly, the characteristic absorption peaks of T-MIPs and T-NIPs were different from the previous work, which might well be owing to that the imprinted layer was grafted onto the surface of HPS. The absorption at 2956 and $2987 \mathrm{~cm}^{-1}$ could be assigned to the stretching vibration of saturated $\mathrm{C}-\mathrm{H}$ bond while $1461 \mathrm{~cm}^{-1}$ could be the bending vibration. The strong absorption at $1735 \mathrm{~cm}^{-1}$ might well be the stretching vibration of $\mathrm{C}=\mathrm{O}$ in aldehyde. The strong absorption at 1156 and $1255 \mathrm{~cm}^{-1}$ could be attributed to the stretching vibration of $\mathrm{C}-\mathrm{N}$ bond. The above peaks proved the imprinted polymers containing pyridine and amide were grafted onto the surface of HPS successfully. Besides, the stretching vibration of $\mathrm{C}=\mathrm{C}$ bond in alkene at $1666 \mathrm{~cm}^{-1}$ for T-MIPs was stronger than that for T-NIPs, which could just indicate that the template molecules well interacted with the compound dual functional monomers in the T-MIPs.

\subsection{Binding studies of T-MIPS}

The binding ability of T-MIPs was investigated by performing the static and dynamic binding studies. Fig. 3a displays the static 
a

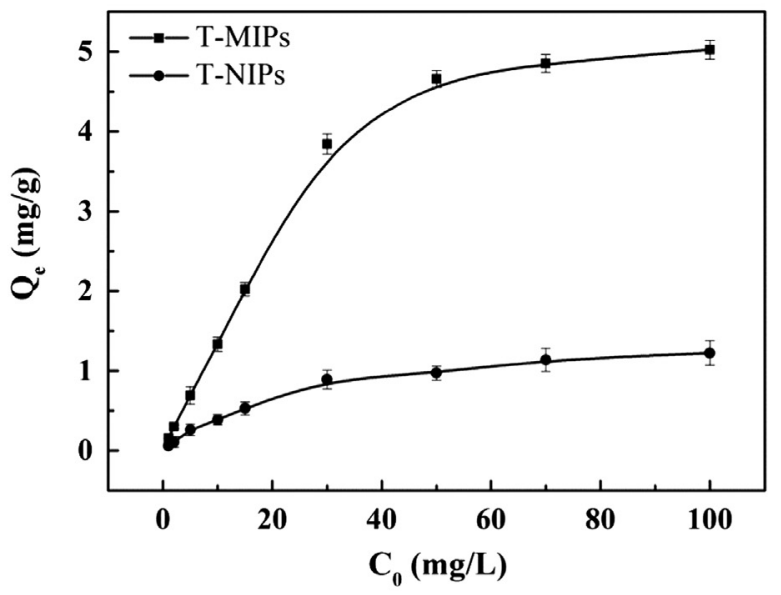

b
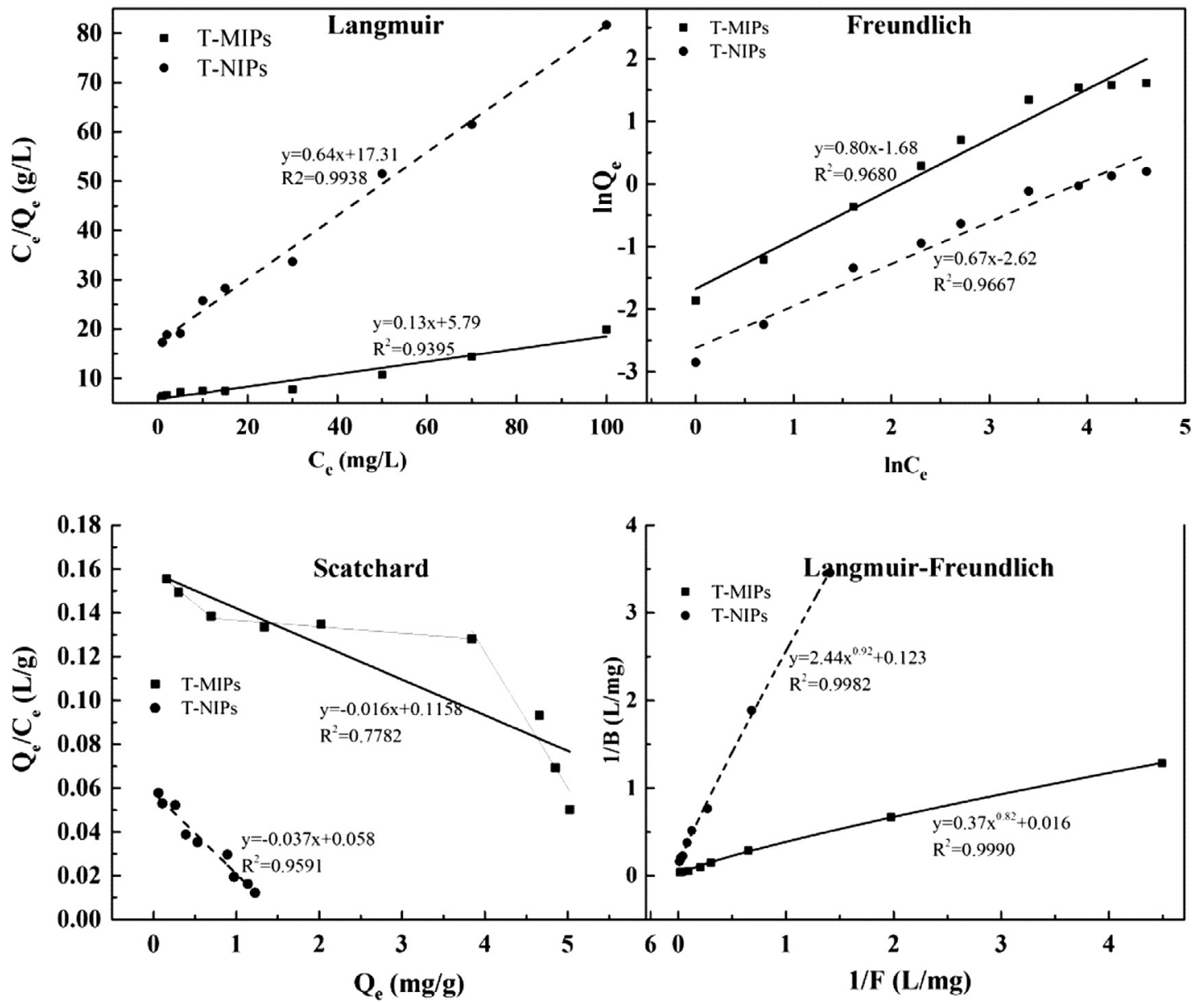

Fig. 3. (a) Static adsorption isotherms and (b) isotherms fitting of T-MIPs and T-NIPs.

binding isotherms of BPA by T-MIPs and T-NIPs. As shown, the adsorption capacity for BPA increased quickly at first and then slowed down with its initial concentration increasing. And the adsorption became stable and reached equilibrium at the concentration of $50 \mathrm{mg} / \mathrm{L}$, indicating that the recognition sites were almost completely occupied by BPA when exceeding the equilibrium concentration. T-NIPs displayed the same trend as T-MIPs but lower adsorption amounts. The maximum binding capacities of T-MIPs and T-NIPs were 5.03 and $1.22 \mathrm{mg} / \mathrm{g}$, respectively, and thereby the imprinted factor $(\alpha)$ obtained was of 4.11 . Moreover, the adsorption process is further studied by four classical isotherm models, Langmuir, Freundlich, Scatchard and LangmuirFreundlich, as shown in Fig. 3b. Their corresponding equations and parameters for adsorption of BPA onto the T-MIPs and T-NIPs are listed in Table S5. It can be seen that the Langmuir-Freundlich model is quite suitable to the adsorption, with the correlation coefficient of 0.9990 . The related parameters comparison of T-MIPs with T-NIPs suggested an excellent imprinting effect owing to the presence of a large number of specific binding sites on the T-MIPs. 
Fig. 4a shows that the adsorption amounts of BPA increase very fast in the initial $60 \mathrm{~min}$ and then slow down gradually to an equilibrium state. The dynamic curve of T-NIPs shared the same trend as T-MIPs but lower adsorption amounts. Meanwhile, four models of the pseudo-first-order, pseudo-second-order, Elovich and intraparticle diffusion models were employed for fitting analysis, as shown in Fig. 4b. As shown, the pseudo-secondorder model could better describe the time effect on the adsorption system, i.e., predict the kinetic process, than other kinetic models, which provided the highest correlation coefficient of
0.9850 as listed in Table S6. Thus, the dynamic adsorption could be deduced to follow the pseudo-second-order kinetics model.

The effects of temperature on the adsorption capacity were investigated for both T-MIPs and T-NIPs at different temperatures, such as 20,33 , and $50{ }^{\circ} \mathrm{C}$. Fig. 5 a shows the adsorption capacities of $\mathrm{BPA}$ at five different concentrations along with the temperature change. As observed, the adsorption amounts of T-MIPs at 20 and $33^{\circ} \mathrm{C}$ were similar and much larger than those at $50{ }^{\circ} \mathrm{C}$ for T-MIPs, which revealed that the material was sensitive to temperature. We can conclude the trend that the adsorption amounts would

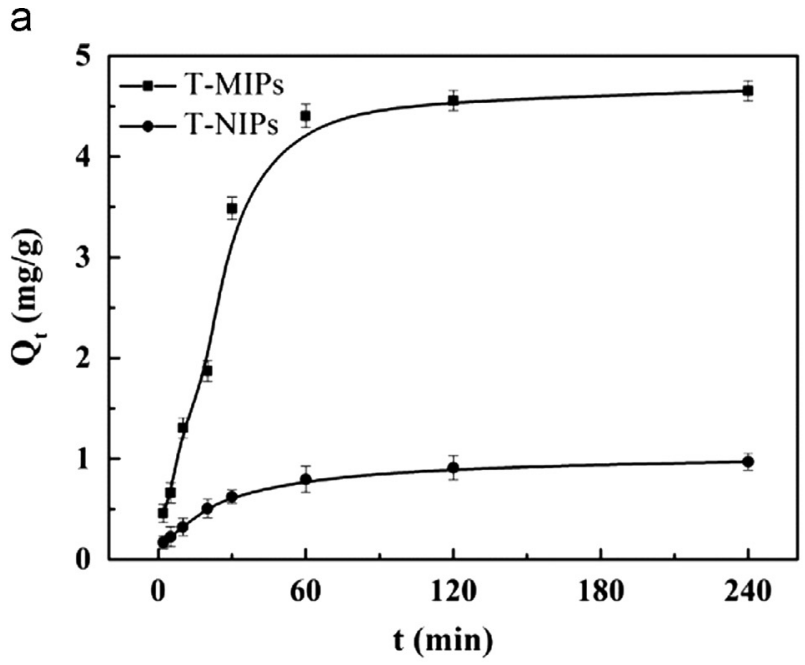

b

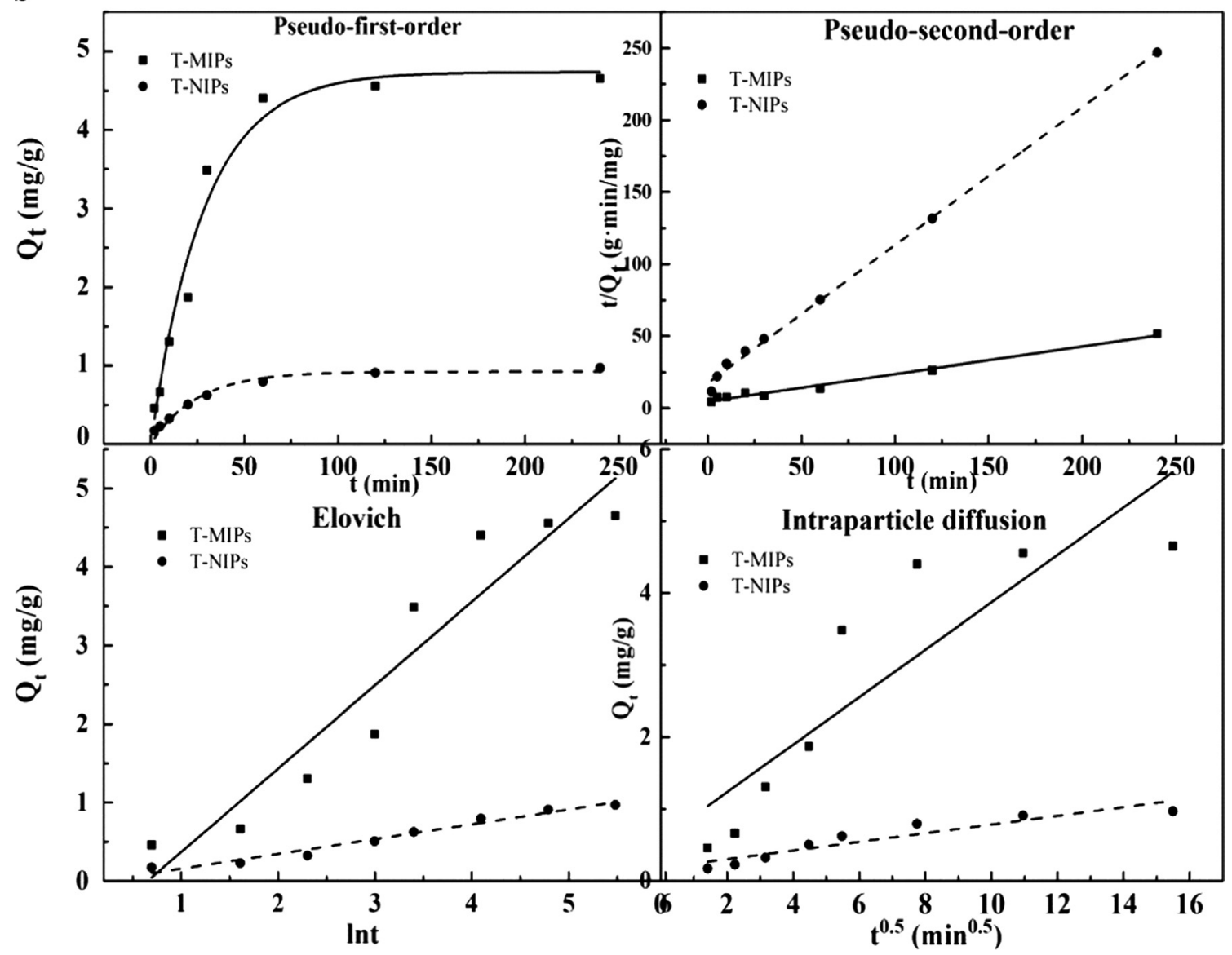

Fig. 4. (a) Dynamic adsorption curves and (b) kinetic fitting of T-MIPs and T-NIPs. 
a

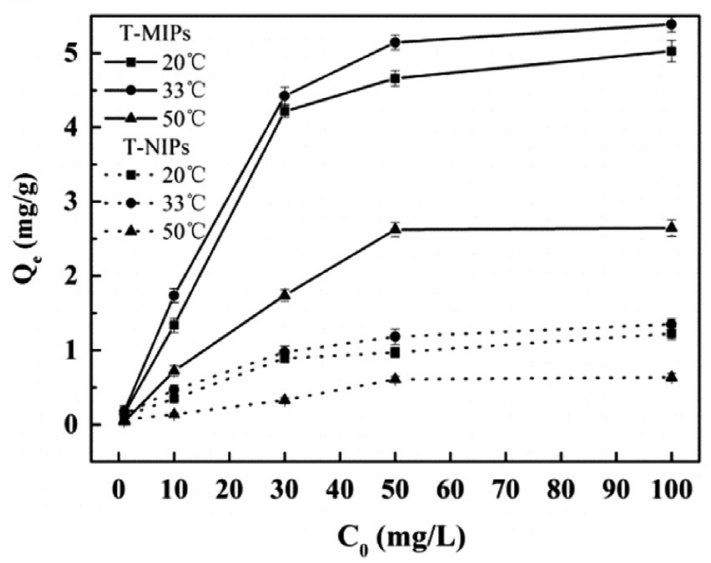

C

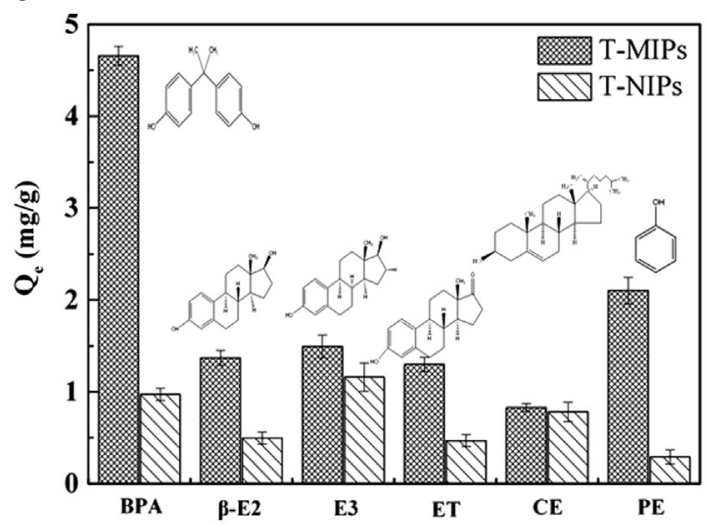

b

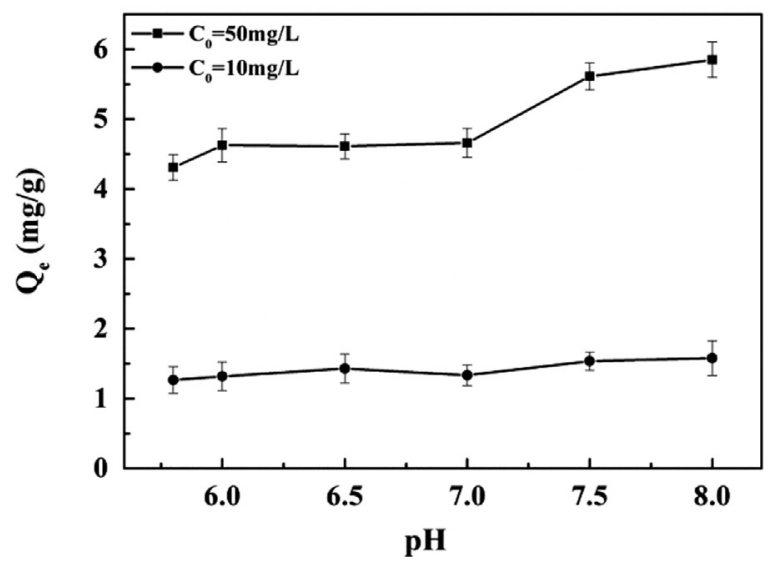

d

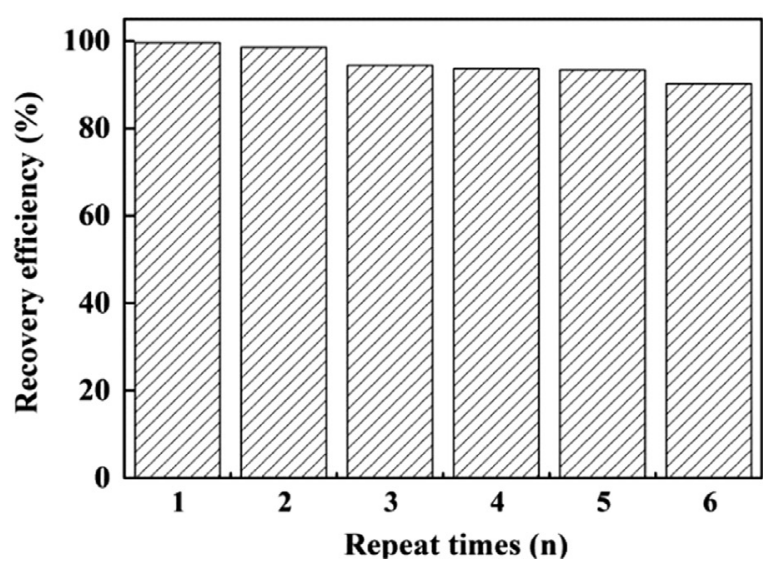

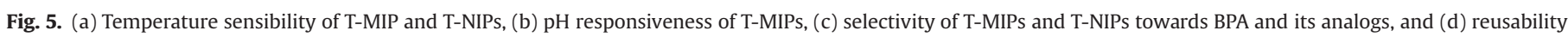
of T-MIPs.

increase slightly along with the rise of temperature under LCST $\left(33^{\circ} \mathrm{C}\right.$ ) and decrease fast above LCST. T-NIPs had the same trend but with smaller adsorption amounts, indicating the thermosensitive monomer played an important role. When the solution temperature is above the LCST, the polymers become hydrophobic and contractive due to the suppression of hydrogen-bonding interaction induced by the thermal phase transition [24,32]. So, by virtue of the synergy interaction of dual functional monomers [32,33], NIPAM and VP, the obtained T-MIPs could effectively control the adsorption amounts of BPA by adjusting the solution temperature, which exhibited high adsorption capacity below LCST (stretched state) and low adsorption capacity above LCST (contractive state). Therefore, considering the conventional experimental environment, $20^{\circ} \mathrm{C}$ was chosen for the following work.

Meanwhile, the influence of different $\mathrm{pH}$ values (5.8, 6.0, 6.5, 7.0, 7.5 and 8.0) on the binding capacities of T-MIPs was studied. Fig. 5b depicts the adsorption amounts of BPA changing along with different solution $\mathrm{pH}$ values at 10 and $50 \mathrm{mg} / \mathrm{L}$, respectively. As shown, the influence of $\mathrm{pH}$ on the adsorption capacity was slight and a little bigger capacity was obtained at 50 than at $10 \mathrm{mg} / \mathrm{L}$. The adsorption amount was slightly higher in an alkaline environment which might be due to the better solubility of BPA in alkaline solution. As a whole, the T-MIPs were not $\mathrm{pH}$ dependent.

Moreover, five similarly structured PEEs to BPA, including $\beta$-E2, $\mathrm{E} 3, \mathrm{ET}, \mathrm{PE}$ and $\mathrm{CE}$, were used to investigate the selective recognition ability of the T-MIPs at $20^{\circ} \mathrm{C}$. As shown in Fig. 5c, the T-MIPs have excellent recognition ability towards BPA, and the selectivity factors $(\beta)$ are between 2.22 and 5.61. However, for T-NIPs, the adsorption amounts of the six PEEs were all lower with no obvious regularity, revealing that the T-NIPs could not selectively adsorb BPA.

\subsection{Applications of T-MIPS as SPE adsorbents to seawater and yogurt samples}

Based on the above results, the T-MIPs could be employed as SPE adsorbents for selective recognition and extraction of BPA. In order to investigate the practical applicability of the T-MIPs, seawater and yogurt samples were treated and prepared by SPE using T-MIPs at $20{ }^{\circ} \mathrm{C}$ followed by HPLC-UV analysis. As listed in Table 1, high recoveries of $94.83-98.47 \%$ with the relative standard deviations (RSD) of $2.72-3.21 \%$ are obtained for the seawater samples, as well as $97.07-98.24 \%$ with the RSD of $2.82-3.16 \%$ for the yogurt samples, spiked with BPA at three concentration levels. On the contrary, the T-NIPs presented remarkably reduced recoveries of $37.83-68.63 \%$ (Table 1). Their corresponding chromatograms are shown in Fig. 6. All the results indicated that the T-MIPs were ideal candidates for SPE, and thereby the T-MIPs-SPE was potentially applicable for highly efficient separation, extraction and determination of trace BPA in real samples.

In the real samples, favorable limit of detection (LOD, $S / N=3$ ) and limit of quantitation (LOQ, $S / N=10$ ) for BPA by using the T-MIPs-SPE were obtained to be 11.14 and $33.42 \mu \mathrm{g} / \mathrm{L}$, respectively. The values meet with the requirement of trace analysis, and the method can reach the required detectability for BPA in environmental water investigations and food safety monitoring to some 
Table 1

Recoveries of BPA in seawater and yogurt samples using T-MIPs and T-NIPs as adsorbents for SPE at $20^{\circ} \mathrm{C}$ followed by HPLC-UV determination.

\begin{tabular}{|c|c|c|c|c|c|}
\hline \multirow[t]{2}{*}{ Samples } & \multirow{2}{*}{$\begin{array}{l}\text { Added } \\
(\mathrm{mg} / \mathrm{L})\end{array}$} & \multicolumn{2}{|l|}{ T-MIPs } & \multicolumn{2}{|l|}{ T-NIPs } \\
\hline & & $\begin{array}{l}\text { Found } \\
(\mathrm{mg} / \mathrm{L})\end{array}$ & $\begin{array}{l}\text { Recovery } \pm \text { RSD } \\
(\%)\end{array}$ & $\begin{array}{l}\text { Found } \\
(\mathrm{mg} / \mathrm{L})\end{array}$ & $\begin{array}{l}\text { Recovery } \pm \text { RSD } \\
(\%)\end{array}$ \\
\hline \multirow[t]{3}{*}{ Seawater } & 1 & 0.97 & $96.70 \pm 2.84$ & 0.59 & $59.08 \pm 2.55$ \\
\hline & 5 & 4.92 & $98.47 \pm 3.21$ & 2.68 & $53.62 \pm 2.22$ \\
\hline & 20 & 18.97 & $94.83 \pm 2.72$ & 13.72 & $68.63 \pm 3.59$ \\
\hline \multirow[t]{3}{*}{ Yogurt } & 1 & 0.97 & $97.07 \pm 3.16$ & 0.38 & $37.83 \pm 3.69$ \\
\hline & 5 & 4.91 & $98.24 \pm 2.93$ & 2.48 & $49.64 \pm 2.54$ \\
\hline & 20 & 19.62 & $98.10 \pm 2.82$ & 10.67 & $53.37 \pm 2.05$ \\
\hline
\end{tabular}

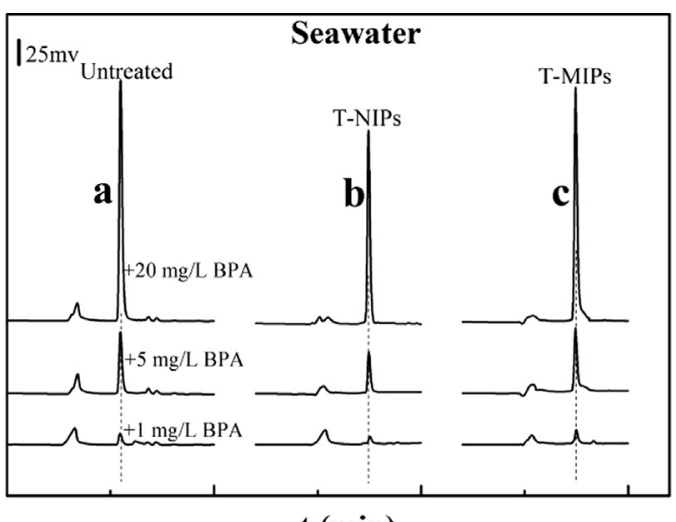

$\mathbf{t}(\min )$

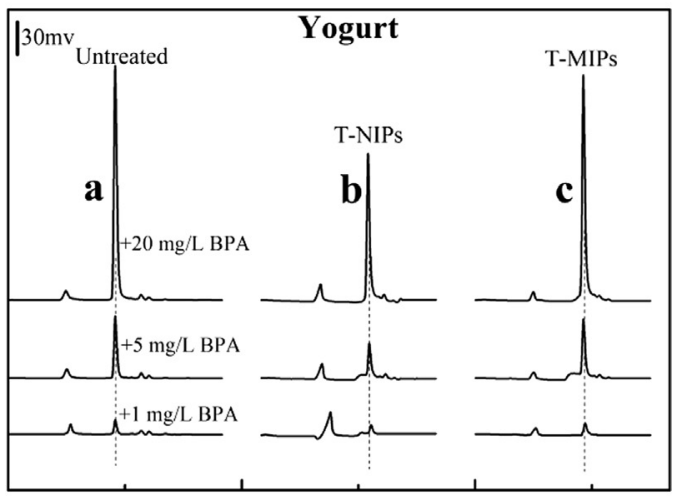

t (min)

Fig. 6. Chromatograms of seawater and yogurt samples spiked with 1,5 and $20 \mathrm{mg} / \mathrm{L}$ BPA untreated (a), with T-NIPs extraction (b) and with T-MIPs extraction (c) at $20^{\circ} \mathrm{C}$.

extent. In addition, reusability of the T-MIPs was evaluated, which has a great cost benefit on extending their applications. As shown in Fig. 5d, the recovery remains $90.2 \%$ after six adsorptiondesorption cycles. Consequently, the attained T-MIPs were demonstrated excellently applicable for reuse without remarkable decrease in their adsorption capacities for BPA. Therefore, the simple, rapid, reliable and reusable T-MIPs proved practically feasible for highly selective and sensitive BPA analysis in complicated matrices.

\section{Conclusions}

In summary, the design and synthesis of thermosensitive MIPs materials for selective recognition and controlled adsorption and release of BPA are demonstrated in this study, via synergy of dual functional monomers. The morphology and structure characterization results clearly confirmed that T-MIPs were successfully prepared with high polymerization efficiency. And the T-MIPs presented high adsorption capacity, fast binding kinetics and good selectivity as well as good stability and reusability. The adsorption behavior of BPA onto T-MIPs obeyed the Langmuir-Freundlich isotherm and the pseudo-second-order kinetic models. Satisfactory recoveries in spiked seawater and yogurt samples proved that the T-MIPs based SPE was a feasible way to rapid selective removal of BPA from complicated matrices. More importantly, the smart materials exhibited good thermosensitivity, which could bring the most ideal performances at below or around LCST. The whole system of recognition, enrichment, separation and release of pollutants can be recycled, so the temperature regulation system is convenient, cost-effective and environment friendly, which provides a new strategy for pollution monitoring and abatement. We believe that it may open up new opportunities to explore various smart SR-MIPs materials for bright perspectives. Our efforts along this line are currently underway.

\section{Acknowledgments}

This work was financially supported by the National Natural Science Foundation of China (21105117, 31160317, 21201098 and 21275158).

\section{Appendix A. Supporting information}

Supplementary data associated with this article can be found in the online version at http://dx.doi.org/10.1016/j.talanta.2014.06.055.

\section{References}

[1] L.Q. Song, W. Xia, Z. Zhou, Y.Y. Li, Y. Lin, J. Wei, Z.Z. Wei, B. Xu, J. Shen, W.Y. Li, S.Q. Xu, J. Endocrinol. 215 (2012) 303-311.

[2] Y.Y. Wen, J.H. Li, J.S. Liu, W.H. Lu, J.P. Ma, L.X. Chen, Anal. Bioanal. Chem. 405 (2013) 5843-5852.

[3] S. Flint, T. Markle, S. Thompson, E. Wallace, J. Environ. Manag. 104 (2012) $19-34$.

[4] V. Migeot, A. Dupuis, A. Cariot, M. Albouy-Llaty, F. Pierre, S. Rabouan, Environ. Sci. Technol. 47 (2013) 13791-13797.

[5] L.N. Vandenberg, P.A. Hunt, J.P. Myers, F.S. vom Saal, Rev. Environ. Health 28 (2013) 37-58.

[6] A. Ballesteros-Gómez, S. Rubio, D.J. Pérez-Bendito, J. Chromatogr. A 1216 (2009) 449-469.

[7] Y. Li, J.L. Liu, Int. J. Environ. Anal. Chem. 90 (2010) 880-890.

[8] M.J. Garber, M. Gill, Y.S. Hua, D. Jenke, J. Chromatogr. Sci. 49 (2011) 214-220.

[9] E. Magi, M.D. Carro, C. Liscio, Anal. Bioanal. Chem. 397 (2010) 1335-1345.

[10] J.H. Li, X.L. Song, Scientific J. Environ. Pollu. Prot. 2 (2013) 50-58.

[11] J.M.L. Martínez, M.F.L. Denis, L.R. Denaday, V.C. Dall'Orto, Talanta 80 (2009) 789-796.

[12] L.X. Chen, S.F. Xu, J.H. Li, Chem. Soc. Rev. 40 (2011) 2922-2942.

[13] X.H. Wang, L.L. Chen, X.J. Xu, Y.Z. Li, Anal. Bioanal. Chem. 401 (2011) $1423-1432$.

[14] J. Li, X.B. Zhang, Y.X. Liu, H.W. Tong, Y.P. Xu, S.M. Liu, Talanta 117 (2013) $281-287$.

[15] L.W. Xia, R. Xie, X.J. Ju, W. Wang, Q.M. Chen, L.Y. Chu, Nat. Commun. 4 (2013) 2226-2236.

[16] H.L. Peng, R.C. Dong, S.Q. Wang, Z. Zhang, M. Luo, C.Q. Bai, Q. Zhao, J.H. Li, L.X. Chen, H. Xiong, Inter. J. Pharm. 446 (2013) 153-159.

[17] S.F. Xu, H.Z. Lu, X.W. Zheng, L.X. Chen, J. Mater. Chem. C 1 (2013) 4406-4422.

[18] Y. Ma, Y. Zhang, M. Zhao, X. Guo, H. Zhang, Chem. Commun. 48 (2012) 6217-6219.

[19] S.F. Xu, J.H. Li, X.L. Song, J.S. Liu, H.Z. Lu, L.X. Chen, Anal. Methods 5 (2013) $124-133$.

[20] N. Griffete, H. Frederich, A. Maitre, S. Ravaine, M.M. Chehimi, C. Mangeney, Langmuir 28 (2011) 1005-1012.

[21] J. Liu, W. Wang, Y. Xie, Y. Huang, Y. Liu, X. Liu, Y. Chen, J. Mater. Chem. 21 (2011) 9232-9238.

[22] Z. Lin, W. Cheng, Y. Li, Z. Liu, X. Chen, C. Huang, Anal. Chim. Acta 720 (2012) 71-76.

[23] B.J. Lu, M.C. Liu, H.J. Shi, X.F. Huang, G.H. Zhao, Electroanalysis 25 (2013) 771-779. 
[24 ] X.J. Li, B.L. Zhang, W. Li, X.F. Lei, X.L. Fan, L. Tian, H.P. Zhang, Q.Y. Zhang, Biosens. Bioelectron. 51 (2014) 261-267.

[25] M. Fernanda, B. Coutinho, V.G. Teixeira, C.C.R. Barbosa, J. Appl. Polym. Sci. 67 (1998) 781-787.

[26] S.F. Xu, L.X. Chen, J.H. Li, W. Qin, J.P. Ma, J. Mater. Chem. 21 (2011) $12047-12053$

[27] Y. Ren, W. Ma, J. Ma, Q. Wen, J. Wang, F. Zhao, J. Colloid Interface Sci. 367 (2012) 355-361.

[28] T. Barri, T. Trtić-Petrović, M. Karlsson, J.Å. Jönsson, J. Pharm. Biomed. Anal. 48 (2008) 49-56.
[29] M.C. Cela-Pérez, M.M. Castro-López, A. Lasagabáster-Latorre, J.M. López-Vilariño, M.V. González-Rodríguez, L.F. Barral-Losada, Anal. Chim. Acta 706 (2011) $275-284$.

[30] J. Zhu, S. Wei, H. Gu, S.B. Rapole, Q. Wang, Z. Luo, Z. Guo, Environ. Sci. Technol. 46 (2011) 977-985.

[31] X.Y. Wang, Q. Kang, D.Z. Shen, Z. Zhang, J.H. Li, L.X. Chen, Talanta 124 (2014) 7-13.

[32] U. Athikomrattanakul, N. Gajovic-Eichelmann, F.W. Scheller, Anal. Chem. 83 (2011) 7704-7711.

[33] X.Q. Cai, J.H. Li, Z. Zhang, F.F. Yang, R.C. Dong, L.X. Chen, ACS Appl. Mater. Interfaces 6 (2014) 305-313. 